\title{
A Study on the Farm Asset Structures, Cropping Pattern and Cropping Intensity of Sample Farms in Ghazipur District of Eastern Uttar Pradesh, India
}

\author{
Punam Kushwaha ${ }^{1}$, Harendra Pratap Singh Choudhri ${ }^{1}$, G.P. Singh ${ }^{1}$, \\ Ashutosh Kumar Ranjan ${ }^{1}$ and Abhineet ${ }^{2}$
}

\author{
${ }^{I}$ Department of Agricultural Economics, N.D.U.A \& T. Kuamraganj Faizabad (U.P.), India \\ ${ }^{2}$ Department of Agronomy, N.D.U.A \& T. Kuamraganj Faizabad (U.P.), India
}

*Corresponding author

\begin{tabular}{|c|c|}
\hline & $\mathbf{T} \mathbf{R}$ \\
\hline Keywords & \multirow{3}{*}{$\begin{array}{l}\text { Keeping in view the importance of the vegetable crops in nutritional security and } \\
\text { generating the income and employment to the farm population a study on Economic } \\
\text { analysis of tomato in Ghazipur district Eastern Uttar Pradesh was conducted in agriculture } \\
\text { year 2016-2017. Stratified purposive cum random sampling technique was applied to } \\
\text { select the sample respondents' primary data were collected through interview method. } \\
\text { Tabular and function analysis was done to present the result. Overall average size of } \\
\text { holding was } 1.125 \text { ha. Which were } 0.679 \text { ha, } 1.500 \text { ha and } 2.620 \text { ha. at marginal, small and } \\
\text { medium size of farms respectively. Per farm investment was inversely related with size of } \\
\text { holding. The paddy, wheat, tomato and maize, were the main crops of cropping pattern, } \\
\text { cropping intensity was highest on marginal farms followed by small and medium size of } \\
\text { farms. It shows that marginal farmers were more attentive about better utilization of their } \\
\text { tinny land holding. }\end{array}$} \\
\hline Article I & \\
\hline & \\
\hline
\end{tabular}

\section{Introduction}

Tomato is one of the most important vegetables crops of the world with $3^{\text {rd }}$ ranking in area and production. Tomato is one of the most popular vegetable of great commercial and nutritional value. Tomato is grown and consumed by the people, around the world. It is a warm season crop. It is also grown as an off season vegetable in hilly area of India and farmers earn enough income by supplying their produce in the plains from June to September. In our country, huge quantities of tomato are utilized to produce, soup, juice, ketchup, puree, paste and powder. It contents
94 per cent moisture, 0.9 per cent protein, 0.2 per cent fat, 0.8 per cent fibre, 3.4 per cent carbohydrates and rich source of vitamin $c$. Presence of vitamin c, variety of colour and flavors in tomato, makes it popular vegetable among the public. Due to good keeping quality tomato can be preserved and available in the market round the year (A Handbook of Vegetable science).

Tomato fruits mature at green stage could be stored successfully at $10-12^{\circ} \mathrm{C}$ in polyethylene bags of 100 gauge thickness for 4-5 weeks storage life of tomato could be increased by keeping in evaporative cool storage (zero 
energy cool chamber). The cool chamber has been found effective in maintaining fruit acceptability for longer period and minimum weight loss considerably. Higher yield is of no importance if the farmer does not get the remunerative price for his produce. Thus the marketing assumes significant importance to the farmer for getting higher income from the vegetable cultivation. If a grower wants to make profitable production, he must produce good quality of vegetable to acquire the specified market needs.

Thus the grower's decision to cultivate various varieties of vegetables would largely depend upon the demand and preferences of the consumer's prevailing in the market. Some time vegetable producer are in the lack of contact with the market channel and consequently do not get the fair price of their produce which they obtain after putting a lot of labour and capital. The study of marketing cost and margins is useful both for the producer (seller) and consumer. A reference to the marketing costs and margins would show whether the service of the intermediaries are provided at reasonable cost or not.

Moreover, the study of the marketing margins can be used to fix market functionaries and judge the efficiency of marketing system. There is great variation in prices from lean period to peak period affecting marketing costs \& margins and producer's share in consumer's rupees and ultimately affecting the farmer's income. Thus the farmers, especially marginal and small can increase their income and employment from production of tomato.

For the purpose a study of farm structure, cropping pattern and cropping intensity in Ghazipur District was conducted with the following specific objectives:

To study the distribution of cultivable land in study area.
To study the per farm and per hectare investment on different size of sample farms.

To study the cropping pattern and cropping intensity, on the different size of sample farms.

\section{Materials and Methods}

\section{Sampling technique}

Purposive cum random sampling technique was used to select the 100 respondent, from 5 villeges of Bhanwarkola block of Ghazipur district. For the further study all selected sample farmers were grouped in three categories of marginal, small and medium. To justify the representation of all category of farmers proportionate random sampling technique was applied. A sum of 31 marginal, 13 small and 6 medium size of sample farms were studied. Details of sampling are presented in Table 1.

\section{Analytical tools}

The data collected from the sample cultivators were analyzed and estimated with certain statistical techniques.

\section{Average}

The simplest and important measures of average which have been used into statistical analysis were the weighted average. The formula used to estimate the average is:

$\bar{X}=\frac{\sum X}{N}$

Weighted avergae $=\frac{\sum \mathrm{W}_{\mathrm{i}} \mathrm{X}_{\mathrm{i}}}{\sum \mathrm{W}_{\mathrm{i}}}$

Where, 
W. A. = Weighted average

$X_{i}=$ Variable

$W_{i=\text { Weights of }} X_{i}$

\section{Functional analysis}

To study the effect of various independent variables on the output, various forms of production function have been dealt.

However, Cobb-Douglas function was found more suitable to the data; therefore it was used for measuring resource use efficiency.

The mathematical form of Cobb-Douglas function is:

$\mathrm{Y}=\mathrm{aX}_{1}{ }^{\mathrm{b} 1} \cdot \mathrm{X}_{2}{ }^{\mathrm{b} 2} \cdot \mathrm{X}_{3}{ }^{\mathrm{b} 3} \cdot \mathrm{X}_{4}{ }^{\mathrm{b} 4} \cdot \mathrm{X}_{5}{ }^{\mathrm{b} 5} \ldots \ldots \ldots \ldots \mathrm{e}^{\mathrm{u}}$

Where,

$\mathrm{Y}=$ per hectare output (Rs.)

$\mathrm{X}_{1}=$ Manure and fertilizers (Rs/ha)

$\mathrm{X}_{2}=$ Total human labour (Rs./ha)

$\mathrm{X}_{3}=\operatorname{seed}(\mathrm{Rs} / \mathrm{ha})$

$\mathrm{X}_{4}=$ Irrigation charge $(\mathrm{Rs} / \mathrm{ha})$

$\mathrm{X}_{5}=$ Plant protection

$\mathrm{a}=$ Constant (intercept)

$\mathrm{e}^{\mathrm{u}}=$ Error and

$b_{1}, b_{2}, b_{3}$ and $b_{4}$ are the production elasticity of the respective input variables.

Cobb-Douglas production function in log form

$\log Y=\log a+b_{1} \log x_{1}+b_{2} \log x_{2}+b_{3} \log x_{3}$ $+\mathrm{b}_{4} \log \mathrm{x}_{4} \ldots \ldots . \mu \log \mathrm{e}$

This formula was used for estimating the parameters of the function based on sample data.

The marginal value product of inputs was estimated by following Formula
$\operatorname{MVP}\left(X_{j}\right)=\frac{b_{j} \bar{Y}}{\overline{X_{j}}}$

Where,

$\mathrm{MVP}=$ marginal value product of $\mathrm{J}^{\text {th }}$ input

$b_{j}=$ Production elasticity with respect to $X_{j}$

$\bar{Y}_{j}=$ Geometric mean of the dependent variable $\mathrm{Y}$

$\bar{X}_{\mathrm{j}}=$ Geometric mean of the independent variable $\mathrm{X}$

Having estimated the elasticity co-efficient, it is desirable to ascertain the reliability of these estimates. The most commonly used " $t$ " test was applied to know, whether ' $b_{j}$ ' is statistically significant from zero or not at some specified probability level.

$$
\text { 't'cal }=\frac{b j}{S . E \text { of bj }}
$$

If calculated ' $t$ ' value is greater than table value of " $t$ " at specified probability level at " $n$ $\mathrm{k}-1$ ' degree of freedom $b_{j}$ is said to be statistically different from zero.

F test was used to test the significance of the regression as a whole.

$\mathrm{F}=\frac{\text { Regression mean square }}{\text { Error mean square }}=\frac{(\mathrm{SSR} / \mathrm{K})}{\Sigma_{\mathrm{e}^{2}}^{2} /(\mathrm{n}-\mathrm{k}-1)}$

Where,

$\mathrm{SSS}=$ sum of square due to regression

$\Sigma \mathrm{e}^{2}=$ sum of square of error term 
M. V.P. of $j^{\text {th }}$ input factor was tested using the formula

$\mathrm{t}=\mathrm{MVP}_{\mathrm{j}} / \mathrm{S}$.E. of $\mathrm{MVP}_{\mathrm{j}}$

S.E. of $\mathrm{MVP}_{\mathrm{j}}=(\overline{\mathrm{Y} / \mathrm{X}})$ standard error of $\mathrm{b}_{\mathrm{j}}$

\section{Results and Discussion}

The result of the presents study as well as relevant discussion has been presented under following sub heads:

\section{The structure of sample farms}

This section deals with the size of farms, farm assets structure, irrigational structure, cropping pattern and cropping intensity.

\section{Land holding area}

The details of land holding area under different size group of sample farms are given in Table 2.

The average size of holding of marginal, small, and medium, farms were found 0.679, 1.500, and 2.620 hectares respectively, and on an overall average size of land holding was estimated as 1.125 hectare.

\section{Farm asset structure on sample farms}

Table 3 presents the per- farm asset structure on sample farms. It is evident from this table that major components of farm asset structure are Buildings, live-stocks and machinery and implements which were constituting 56.37 per cent, 12.31 per cent and 31.31 per cent of total asset value respectively on the basis of overall average. Per farm buildings, major implements and livestock came to Rs.169005.20, Rs. 93881.65 and Rs. 36911.98 respectively. On an average per farm investment was found Rs. 299798.90. The highest investment was recorded on medium farm Rs.477465.35 followed by small Rs 35126.49 and lowest on marginal farms Rs 243048.59 respectively. The per farm investment on farm assets showed the direct relationship with size of holding.

\section{Per hectare investment on different size group of farms}

Investment on different size group of farm on per hectare basis is presented in Table 4. On an overall average per hectare investment was found Rs. 305006.70, which was recorded higher on marginal farms Rs. 357950.80, followed by small Rs. 235417.70 and was lowest on medium farms i.e. 182238.70 respectively.

\section{Cropping pattern}

Cropping pattern presents the area devoted to the various crop during the given period, conventionally in a single year. It indicates the yearly sequence and arrangement of crops grown by farmer in a particular area. The cropping patterns followed by the sample farms are presented in Table 5.

It is obvious from the Table 5 that on an average the highest area was covered under paddy 15.19 per cent followed by wheat 15.19 per cent, tomato 12.44 per cent, maize, 6.67 per cent, cauliflower 5.04 per cent, mustard 4.30 per cent, sugarcane 3.22 per cent, pea 3.19 per cent, cabbage 3.09 per cent, cauliflower 2.99 per cent, gram 2.87 per cent, berseem 2.47 per cent, bottle gourd 2.44 per cent, chilli 2.35 per cent, urd 2.19 per cent, cucumber 2.06 per cent, okra 1.61 per cent watermelon 1.55 per cent, bitter gourd 1.35 per cent, arhar 1.34 per cent, muskmelon 1.30 per cent, bajra 1.25 per cent, brinjal 1.16 per cent, chilli 1.01 per cent and black gram 0.96 of total cropped area on sample farm. Tomato crop was raised by the sample farms after paddy and wheat. 
Table.1 Village wise proportionate selection of sample farmers under different size group of farms

\begin{tabular}{|c|c|c|c|c|c|c|c|c|c|}
\hline \multirow[t]{2}{*}{ SI. No. } & \multirow{2}{*}{$\begin{array}{l}\text { Size of farms } \\
\text { Name of Villages }\end{array}$} & \multicolumn{2}{|c|}{$\begin{array}{l}\text { Marginal } \\
(<1 \text { ha. })\end{array}$} & \multicolumn{2}{|c|}{$\begin{array}{l}\text { Small } \\
\text { (1-2 ha.) }\end{array}$} & \multicolumn{2}{|c|}{$\begin{array}{l}\text { Medium } \\
\text { (2-4 ha.) }\end{array}$} & \multicolumn{2}{|c|}{ Total } \\
\hline & & $\mathrm{P}$ & $\mathrm{S}$ & $\mathrm{P}$ & $\mathrm{S}$ & $\mathrm{P}$ & $\mathrm{S}$ & $\mathrm{P}$ & S \\
\hline 1. & Pakhanpura & 88 & 6 & 46 & 3 & 20 & 1 & 154 & 10 \\
\hline 2. & Hadaria & 96 & 7 & 50 & 4 & 25 & 2 & 171 & 13 \\
\hline 3. & Bhawanrkola & 90 & 6 & 43 & 3 & 18 & 1 & 151 & 10 \\
\hline 4. & Firozpur & 74 & 5 & 35 & 2 & 20 & 1 & 129 & 8 \\
\hline 5. & Jasdevpur & 95 & 7 & 20 & 1 & 8 & 1 & 123 & 9 \\
\hline & Total & 443 & 31 & 194 & 13 & 91 & 6 & 728 & 50 \\
\hline
\end{tabular}

Note: $\mathrm{P}=$ Population and $\mathrm{S}=$ Sample.

Table.2 Average size of holding on different size group of sample farms (ha)

\begin{tabular}{|l|l|c|c|c|}
\hline Sl. No. & Size groups of farmers & No. of farmers & Net cultivated area (ha) & A verage size of farms \\
\hline 1. & Marginal & 31 & $21.049(37.41)$ & 0.679 \\
\hline 2. & Small & 13 & $19.500(34.65)$ & 1.500 \\
\hline 3. & Medium & 6 & $15.720(27.94)$ & 2.620 \\
\hline Grand Total & 50 & $56.269(100)$ & $1.125^{*}$ \\
\hline
\end{tabular}

*Indicate the overall average (Figures in parentheses indicate percentage to total).

Table.3 Per farm investment on different size group of sample farms (Rs.)

\begin{tabular}{|c|c|c|c|c|c|}
\hline \multirow[t]{2}{*}{ S. No. } & \multirow[t]{2}{*}{ Particulars } & \multicolumn{4}{|c|}{ Size of farms } \\
\hline & & Marginal & Small & Medium & Overall average \\
\hline 1. & Buildings & $\begin{array}{c}139811.97 \\
(57.52)\end{array}$ & $\begin{array}{c}196752.72 \\
(55.72)\end{array}$ & $\begin{array}{c}259717.44 \\
(54.39)\end{array}$ & $\begin{array}{c}169005.20 \\
(56.37)\end{array}$ \\
\hline \multirow[t]{3}{*}{ I. } & Residential & $\begin{array}{c}131159.06 \\
(53.96)\end{array}$ & $\begin{array}{c}186288.45 \\
(52.75)\end{array}$ & $\begin{array}{l}250310.64 \\
(52.42)\end{array}$ & $\begin{array}{c}159790.90 \\
(53.29)\end{array}$ \\
\hline & a. Kachcha & $\begin{array}{c}5022.19 \\
(2.07)\end{array}$ & $\begin{array}{c}5230.76 \\
(1.48)\end{array}$ & $\begin{array}{c}4970.41 \\
(1.04)\end{array}$ & $\begin{array}{c}5070.20 \\
(1.69)\end{array}$ \\
\hline & b. $\quad$ Pacca & $\begin{array}{c}126136.87 \\
(51.89)\end{array}$ & $\begin{array}{c}181057.69 \\
(51.27)\end{array}$ & $\begin{array}{c}245340.23 \\
(51.38)\end{array}$ & $\begin{array}{c}154720.70 \\
(51.61)\end{array}$ \\
\hline \multirow[t]{3}{*}{ I. } & Cattle shed & $\begin{array}{c}8652.91 \\
(3.56)\end{array}$ & $\begin{array}{c}10464.27 \\
(2.96)\end{array}$ & $\begin{array}{c}9406.80 \\
(1.97)\end{array}$ & $\begin{array}{c}9214.33 \\
(3.07)\end{array}$ \\
\hline & a. Kachcha & $\begin{array}{l}6970.22 \\
(2.87)\end{array}$ & $\begin{array}{c}4214.28 \\
(1.19)\end{array}$ & $\begin{array}{c}3054.73 \\
(0.63)\end{array}$ & $\begin{array}{c}5783.82 \\
(1.93)\end{array}$ \\
\hline & b. $\quad$ Pacca & $\begin{array}{c}1682.69 \\
(0.69)\end{array}$ & $\begin{array}{c}6249.99 \\
(1.76)\end{array}$ & $\begin{array}{c}6352.07 \\
(1.33)\end{array}$ & $\begin{array}{c}3430.51 \\
(1.14)\end{array}$ \\
\hline 2. & Live stock & $\begin{array}{c}30671.03 \\
(12.62)\end{array}$ & $\begin{array}{c}44084.99 \\
(12.48)\end{array}$ & $\begin{array}{c}53615.38 \\
(11.22)\end{array}$ & $\begin{array}{c}36911.98 \\
(12.31)\end{array}$ \\
\hline$\overline{\mathrm{I}}$. & Milch Animals & $\begin{array}{c}30671.03 \\
(12.61)\end{array}$ & $\begin{array}{c}44084.99 \\
(12.48)\end{array}$ & $\begin{array}{c}53615.38 \\
(11.22)\end{array}$ & $\begin{array}{c}36911.98 \\
(12.31)\end{array}$ \\
\hline a. & Cow & $\begin{array}{c}8001.29 \\
(3.29)\end{array}$ & $\begin{array}{c}11428.57 \\
(3.23)\end{array}$ & $\begin{array}{c}11000.00 \\
(2.30)\end{array}$ & $\begin{array}{c}9252.23 \\
(3.08)\end{array}$ \\
\hline b. & Buffalo & $\begin{array}{c}22342.82 \\
(9.19)\end{array}$ & $\begin{array}{c}31200.00 \\
(8.83)\end{array}$ & $\begin{array}{c}42615.38 \\
(8.92)\end{array}$ & $\begin{array}{c}27078.39 \\
(9.03)\end{array}$ \\
\hline c. & Goat & $\begin{array}{l}326.92 \\
(0.13)\end{array}$ & $\begin{array}{c}1456.42 \\
(0.41)\end{array}$ & $\begin{array}{c}00.00 \\
(00.00)\end{array}$ & $\begin{array}{l}581.36 \\
(0.19)\end{array}$ \\
\hline 3. & $\begin{array}{l}\text { Machinery and } \\
\text { Implements }\end{array}$ & $\begin{array}{c}72565.59 \\
(29.85)\end{array}$ & $\begin{array}{c}112288.78 \\
(31.79)\end{array}$ & $\begin{array}{c}164132.53 \\
(34.37)\end{array}$ & $\begin{array}{c}93881.65 \\
(31.31)\end{array}$ \\
\hline I. & Minor Implements & $\begin{array}{l}365.37 \\
(0.15)\end{array}$ & $\begin{array}{c}415.99 \\
(0.12)\end{array}$ & $\begin{array}{l}491.35 \\
(0.10)\end{array}$ & $\begin{array}{l}393.65 \\
(0.13)\end{array}$ \\
\hline I. & Major Implements & $\begin{array}{c}72200.22 \\
(27.70)\end{array}$ & $\begin{array}{c}111872.79 \\
(3.16)\end{array}$ & $\begin{array}{c}163641.18 \\
(34.27)\end{array}$ & $\begin{array}{c}93488.00 \\
(31.18)\end{array}$ \\
\hline 4. & Grand total & $\begin{array}{c}243048.59 \\
(100)\end{array}$ & $\begin{array}{c}353126.49 \\
(100)\end{array}$ & $\begin{array}{c}477465.35 \\
(100)\end{array}$ & $\begin{array}{l}299798.90 \\
(100)\end{array}$ \\
\hline
\end{tabular}

(Figures in parentheses indicate percentage to per farm to the total cost under each size of samples). 
Table.4 Per hectare investment on different size group of sample farms (Rs.)

\begin{tabular}{|c|c|c|c|c|c|}
\hline \multirow{2}{*}{$\begin{array}{l}\text { S. } \\
\text { No. }\end{array}$} & \multirow[t]{2}{*}{ Particulars } & \multicolumn{4}{|c|}{ Size of farms } \\
\hline & & Marginal & Small & Medium & $\begin{array}{l}\text { Overall } \\
\text { average }\end{array}$ \\
\hline 1. & Buildings & $\begin{array}{c}205908.60 \\
(57.52)\end{array}$ & $\begin{array}{c}131168.50 \\
(55.72)\end{array}$ & $\begin{array}{c}99128.79 \\
(54.39)\end{array}$ & $\begin{array}{c}173662.60 \\
(56.37)\end{array}$ \\
\hline \multirow[t]{3}{*}{ A. } & Residential & $\begin{array}{c}193165.00 \\
(53.96)\end{array}$ & $\begin{array}{c}124192.30 \\
(52.75)\end{array}$ & $\begin{array}{c}95538.41 \\
(52.42)\end{array}$ & $\begin{array}{c}163516.90 \\
(53.29)\end{array}$ \\
\hline & a. Kachcha & $\begin{array}{c}7396.45 \\
(2.07)\end{array}$ & $\begin{array}{c}3487.17 \\
(1.48)\end{array}$ & $\begin{array}{c}1897.10 \\
(1.04)\end{array}$ & $\begin{array}{c}5720.10 \\
(1.69)\end{array}$ \\
\hline & b. Pacca & $\begin{array}{c}185768.60 \\
(51.89)\end{array}$ & $\begin{array}{c}120705.10 \\
(51.27)\end{array}$ & $\begin{array}{c}93641.31 \\
(51.38)\end{array}$ & $\begin{array}{c}157796.80 \\
(51.61)\end{array}$ \\
\hline \multirow[t]{3}{*}{ B. } & Cattle shed & $\begin{array}{c}12743.61 \\
(3.56)\end{array}$ & $\begin{array}{c}6976.18 \\
(2.96)\end{array}$ & $\begin{array}{c}3590.38 \\
(1.97)\end{array}$ & $\begin{array}{c}10145.69 \\
(3.07)\end{array}$ \\
\hline & a. Kachcha & $\begin{array}{c}10265.42 \\
(2.87)\end{array}$ & $\begin{array}{c}2809.52 \\
(1.19)\end{array}$ & $\begin{array}{c}1165.93 \\
(0.63)\end{array}$ & $\begin{array}{c}7234.95 \\
(1.93)\end{array}$ \\
\hline & b. Pacca & $\begin{array}{c}2478.19 \\
(0.69)\end{array}$ & $\begin{array}{c}4166.66 \\
(1.76)\end{array}$ & $\begin{array}{c}2424.45 \\
(1.33)\end{array}$ & $\begin{array}{c}2910.74 \\
(1.14)\end{array}$ \\
\hline 2. & Live stock & $\begin{array}{c}45170.88 \\
(12.62)\end{array}$ & $\begin{array}{c}29389.99 \\
(12.48)\end{array}$ & $\begin{array}{c}20463.89 \\
(11.22)\end{array}$ & $\begin{array}{c}38103.01 \\
(12.31)\end{array}$ \\
\hline A. & Milch Animals & $\begin{array}{l}45170.88 \\
(12.61)\end{array}$ & $\begin{array}{c}29389.99 \\
(12.48)\end{array}$ & $\begin{array}{c}20463.89 \\
(11.22)\end{array}$ & $\begin{array}{l}38103.01 \\
(12.31)\end{array}$ \\
\hline a. & Cow & $\begin{array}{c}11783.93 \\
(3.29)\end{array}$ & $\begin{array}{c}7619.05 \\
(3.23)\end{array}$ & $\begin{array}{c}4198.47 \\
(2.30)\end{array}$ & $\begin{array}{c}9790.81 \\
(3.08)\end{array}$ \\
\hline b. & Buffalo & $\begin{array}{l}32905.48 \\
(9.19)\end{array}$ & $\begin{array}{l}20800.00 \\
(8.83)\end{array}$ & $\begin{array}{c}16265.41 \\
(8.92)\end{array}$ & $\begin{array}{c}27761.25 \\
(9.03)\end{array}$ \\
\hline c. & Goat & $\begin{array}{c}481.47 \\
(0.13)\end{array}$ & $\begin{array}{c}970.95 \\
(0.41)\end{array}$ & $\begin{array}{c}00 \\
(00.00)\end{array}$ & $\begin{array}{c}550.96 \\
(0.19)\end{array}$ \\
\hline 3. & $\begin{array}{l}\text { Machinery and } \\
\text { Implements }\end{array}$ & $\begin{array}{c}106871.30 \\
(29.85)\end{array}$ & $\begin{array}{c}74859.19 \\
(31.79)\end{array}$ & $\begin{array}{c}62646.00 \\
(34.37)\end{array}$ & $\begin{array}{c}93241.09 \\
(31.31)\end{array}$ \\
\hline A. & $\begin{array}{l}\text { Minor } \\
\text { Implements }\end{array}$ & $\begin{array}{c}538.10 \\
(0.15)\end{array}$ & $\begin{array}{c}277.33 \\
(0.12)\end{array}$ & $\begin{array}{l}187.54 \\
(0.10)\end{array}$ & $\begin{array}{l}428.23 \\
(0.13)\end{array}$ \\
\hline B. & $\begin{array}{l}\text { Major } \\
\text { Implements }\end{array}$ & $\begin{array}{c}106333.20 \\
(29.70)\end{array}$ & $\begin{array}{c}74581.86 \\
(3.16)\end{array}$ & $\begin{array}{c}62458.47 \\
(34.27)\end{array}$ & $\begin{array}{c}92812.86 \\
(31.18)\end{array}$ \\
\hline 4. & Grand total & $\begin{array}{l}357950.80 \\
(100)\end{array}$ & $\begin{array}{l}235417.70 \\
\quad(100)\end{array}$ & $\begin{array}{l}182238.70 \\
\quad(100)\end{array}$ & $\begin{array}{l}305006.7 \\
(100)\end{array}$ \\
\hline
\end{tabular}


Table.5 Cropping pattern under different size group of sample farms (ha)

\begin{tabular}{|c|c|c|c|c|c|}
\hline \multirow[t]{2}{*}{ Sl. No. } & \multirow[t]{2}{*}{ Crop } & \multicolumn{3}{|c|}{ Average size of sample farms } & \multirow[t]{2}{*}{ Overall Average } \\
\hline & & Marginal & Small & Medium & \\
\hline A. & Kharif & $\begin{array}{c}0.673 \\
(41.80)\end{array}$ & $\begin{array}{c}1.457 \\
(44.75)\end{array}$ & $\begin{array}{c}2.500 \\
(46.19)\end{array}$ & $\begin{array}{c}1.096 \\
(43.95)\end{array}$ \\
\hline 1. & Tomato & $\begin{array}{c}0.166 \\
(10.31)\end{array}$ & $\begin{array}{c}0.465 \\
(14.28)\end{array}$ & $\begin{array}{c}0.720 \\
(13.30)\end{array}$ & $\begin{array}{c}0.310 \\
(12.44)\end{array}$ \\
\hline 2. & Paddy & $\begin{array}{c}0.220 \\
(13.66)\end{array}$ & $\begin{array}{c}0.480 \\
(14.74)\end{array}$ & $\begin{array}{c}0.980 \\
(18.10)\end{array}$ & $\begin{array}{c}0.379 \\
(15.19)\end{array}$ \\
\hline 3. & Maize & $\begin{array}{l}0.102 \\
(6.34)\end{array}$ & $\begin{array}{l}0.240 \\
(7.31)\end{array}$ & $\begin{array}{l}0.339 \\
(6.26)\end{array}$ & $\begin{array}{l}0.166 \\
(6.67)\end{array}$ \\
\hline 4. & Cauliflower & $\begin{array}{l}0.100 \\
(6.21)\end{array}$ & $\begin{array}{l}0.130 \\
(3.99)\end{array}$ & $\begin{array}{l}0.250 \\
(4.62)\end{array}$ & $\begin{array}{l}0.126 \\
(5.04)\end{array}$ \\
\hline 5. & Brinjal & $\begin{array}{l}0.025 \\
(1.55)\end{array}$ & $\begin{array}{l}0.024 \\
(0.74)\end{array}$ & $\begin{array}{l}0.060 \\
(1.11)\end{array}$ & $\begin{array}{l}0.029 \\
(1.16)\end{array}$ \\
\hline 6. & Bajra & $\begin{array}{l}0.015 \\
(0.93)\end{array}$ & $\begin{array}{c}0.038 \\
(1.17)\end{array}$ & $\begin{array}{l}0.100 \\
(1.85)\end{array}$ & $\begin{array}{l}0.031 \\
(1.25)\end{array}$ \\
\hline 7. & Blackgram & $\begin{array}{l}0.025 \\
(1.55)\end{array}$ & $\begin{array}{l}0.025 \\
(0.77)\end{array}$ & $\begin{array}{l}0.016 \\
(0.29)\end{array}$ & $\begin{array}{l}0.024 \\
(0.96)\end{array}$ \\
\hline 8. & Arhar & $\begin{array}{l}0.020 \\
(1.24)\end{array}$ & $\begin{array}{l}0.065 \\
(1.99)\end{array}$ & $\begin{array}{l}0.035 \\
(0.65)\end{array}$ & $\begin{array}{l}0.034 \\
(1.34)\end{array}$ \\
\hline B. & Rabi & $\begin{array}{c}0.643 \\
(39.94)\end{array}$ & $\begin{array}{c}1.301 \\
(39.96)\end{array}$ & $\begin{array}{c}2.425 \\
(44.80)\end{array}$ & $\begin{array}{c}1.028 \\
(41.22)\end{array}$ \\
\hline 1. & Wheat & $\begin{array}{c}0.220 \\
(13.66)\end{array}$ & $\begin{array}{c}0.480 \\
(14.74)\end{array}$ & $\begin{array}{l}0.980 \\
(18.1)\end{array}$ & $\begin{array}{c}0.379 \\
(15.19)\end{array}$ \\
\hline 2. & Pea & $\begin{array}{l}0.050 \\
(3.11)\end{array}$ & $\begin{array}{l}0.110 \\
(3.28)\end{array}$ & $\begin{array}{l}0.166 \\
(3.07)\end{array}$ & $\begin{array}{l}0.080 \\
(3.19)\end{array}$ \\
\hline 3. & Gram & $\begin{array}{l}0.050 \\
(3.11)\end{array}$ & $\begin{array}{c}0.093 \\
(2.85)\end{array}$ & $\begin{array}{l}0.140 \\
(2.58)\end{array}$ & $\begin{array}{l}0.072 \\
(2.87)\end{array}$ \\
\hline 4. & Potato & $\begin{array}{l}0.043 \\
(2.67)\end{array}$ & $\begin{array}{l}0.098 \\
(3.01)\end{array}$ & $\begin{array}{l}0.153 \\
(2.83)\end{array}$ & $\begin{array}{l}0.071 \\
(2.83)\end{array}$ \\
\hline 5. & Mustard & $\begin{array}{l}0.065 \\
(4.04)\end{array}$ & $\begin{array}{l}0.166 \\
(5.09)\end{array}$ & $\begin{array}{l}0.198 \\
(3.66)\end{array}$ & $\begin{array}{l}0.107 \\
(4.30)\end{array}$ \\
\hline 6. & Barseem & $\begin{array}{l}0.036 \\
(2.24)\end{array}$ & $\begin{array}{l}0.088 \\
(2.70)\end{array}$ & $\begin{array}{l}0.136 \\
(2.51)\end{array}$ & $\begin{array}{l}0.062 \\
(2.47)\end{array}$ \\
\hline 7. & Chilli & $\begin{array}{l}0.011 \\
(0.68)\end{array}$ & $\begin{array}{l}0.030 \\
(0.92)\end{array}$ & $\begin{array}{l}0.098 \\
(1.81)\end{array}$ & $\begin{array}{l}0.026 \\
(1.06)\end{array}$ \\
\hline 8. & Sugarcane & $\begin{array}{l}0.025 \\
(1.55)\end{array}$ & $\begin{array}{l}0.140 \\
(4.30)\end{array}$ & $\begin{array}{l}0.236 \\
(4.36)\end{array}$ & $\begin{array}{l}0.080 \\
(3.22)\end{array}$ \\
\hline 9. & Cabbage & $\begin{array}{l}0.068 \\
(4.22)\end{array}$ & $\begin{array}{l}0.046 \\
(1.41)\end{array}$ & $\begin{array}{l}0.193 \\
(3.56)\end{array}$ & $\begin{array}{l}0.077 \\
(3.09)\end{array}$ \\
\hline 10. & Cauliflower & $\begin{array}{l}0.075 \\
(4.66)\end{array}$ & $\begin{array}{l}0.050 \\
(1.54)\end{array}$ & $\begin{array}{l}0.125 \\
(2.31)\end{array}$ & $\begin{array}{l}0.075 \\
(2.99)\end{array}$ \\
\hline C. & Zaid & $\begin{array}{c}0.294 \\
(18.26)\end{array}$ & $\begin{array}{c}0.498 \\
(15.29)\end{array}$ & $\begin{array}{l}0.488 \\
(9.02)\end{array}$ & $\begin{array}{c}0.370 \\
(14.85)\end{array}$ \\
\hline 1. & Bitter gourd & $\begin{array}{l}0.015 \\
(0.93)\end{array}$ & $\begin{array}{l}0.065 \\
(1.99)\end{array}$ & $\begin{array}{l}0.063 \\
(1.16)\end{array}$ & $\begin{array}{l}0.034 \\
(1.35)\end{array}$ \\
\hline 2. & Okra & $\begin{array}{l}0.026 \\
(1.62)\end{array}$ & $\begin{array}{c}0.053 \\
(1.628)\end{array}$ & $\begin{array}{l}0.085 \\
(1.57)\end{array}$ & $\begin{array}{l}0.040 \\
(1.61)\end{array}$ \\
\hline 3. & Muskmelon & $\begin{array}{l}0.026 \\
(1.66)\end{array}$ & $\begin{array}{l}0.038 \\
(1.17)\end{array}$ & $\begin{array}{l}0.053 \\
(0.98)\end{array}$ & $\begin{array}{l}0.032 \\
(1.30)\end{array}$ \\
\hline 4. & Watermelon & $\begin{array}{l}0.028 \\
(1.74)\end{array}$ & $\begin{array}{l}0.053 \\
(1.63)\end{array}$ & $\begin{array}{l}0.062 \\
(1.14)\end{array}$ & $\begin{array}{l}0.039 \\
(1.55)\end{array}$ \\
\hline 6. & Urd & $\begin{array}{l}0.033 \\
(2.05)\end{array}$ & $\begin{array}{l}0.090 \\
(2.76)\end{array}$ & $\begin{array}{l}0.090 \\
(1.66)\end{array}$ & $\begin{array}{l}0.055 \\
(2.19)\end{array}$ \\
\hline 7. & Bottle gourd & $\begin{array}{l}0.050 \\
(3.11)\end{array}$ & $\begin{array}{l}0.098 \\
(3.01)\end{array}$ & $\begin{array}{l}0.037 \\
(0.68)\end{array}$ & $\begin{array}{l}0.061 \\
(2.44)\end{array}$ \\
\hline 8. & Cucumber & $\begin{array}{l}0.053 \\
(3.29)\end{array}$ & $\begin{array}{l}0.053 \\
(1.63)\end{array}$ & $\begin{array}{l}0.039 \\
(0.72)\end{array}$ & $\begin{array}{l}0.051 \\
(2.06)\end{array}$ \\
\hline 9. & Chilli & $\begin{array}{l}0.063 \\
(3.91)\end{array}$ & $\begin{array}{l}0.048 \\
(1.47)\end{array}$ & $\begin{array}{l}0.059 \\
(1.09)\end{array}$ & $\begin{array}{l}0.059 \\
(2.35)\end{array}$ \\
\hline & $\begin{array}{l}\text { Gross cropped } \\
\text { area }\end{array}$ & $\begin{array}{l}1.610 \\
(100)\end{array}$ & $\begin{array}{l}3.256 \\
(100)\end{array}$ & $\begin{array}{l}5.413 \\
(100)\end{array}$ & $\begin{array}{l}2.494 \\
(100)\end{array}$ \\
\hline
\end{tabular}

Figures in parentheses indicate the percentage area under crops. 
Table.6 Cropping intensity of different size group of farms

\begin{tabular}{|l|l|l|l|l|l|}
\hline $\begin{array}{l}\text { S. } \\
\text { No. }\end{array}$ & $\begin{array}{l}\text { Size group of } \\
\text { farms }\end{array}$ & $\begin{array}{l}\text { No. of } \\
\text { farms }\end{array}$ & $\begin{array}{l}\text { Net cultivated } \\
\text { area (ha) }\end{array}$ & $\begin{array}{l}\text { Gross cropped } \\
\text { area (ha) }\end{array}$ & $\begin{array}{l}\text { Cropping } \\
\text { intensity (\%) }\end{array}$ \\
\hline 1. & Marginal & 31 & 0.679 & 1.610 & $\mathbf{2 3 7 . 1 1}$ \\
\hline 2. & Small & 13 & 1.500 & 3.256 & $\mathbf{2 1 7 . 0 7}$ \\
\hline 3. & Medium & 6 & 2.620 & 5.413 & $\mathbf{2 0 6 . 6 0}$ \\
\hline & Average & $\mathbf{5 0}$ & $\mathbf{1 . 1 2 5}$ & $\mathbf{2 . 4 9 4}$ & $\mathbf{2 2 1 . 6 9 *}$ \\
\hline
\end{tabular}

*Indicate overall average percentage of cropping intensity

The gross cultivated area was higher in the kharif followed by rabi season and less in the Zaid season on all farm situations. It is also clear from Table 5 that tomato 12.44 per cent in the kharif season of total cropped area.

Small farmers devoting highest area for cultivation of tomato 14.28 per cent, followed by medium 13.30 per cent, and marginal 10.31 per cent, respectively of their total cultivated area.

\section{Cropping intensity}

The details of cropping intensity are given in the Table 6.

It is revealed from Table 6 reveals that the overall average cropping intensity on sample farms was 221.69 per cent which was found highest on marginal farms 237.11 per cent followed by small 217.07 per cent, and medium 206.60 per cent respectively. Cropping intensity was inversely related to size of farms. Highest cropping intensity on marginal sample form supported the engagement of family labour in their own crop production on keeping in view the better utilization of their tinny land holding.

\section{References}

Kumar Narendra, M. Srivastva, A. K. (2009). Off-season vegetable-based cropping sequence under protected cultivation in mid-hills of north-western Himalayan region. Indian Journal of Agricultural Sciences. 79: 7, 531-534.

Sharma

Ravinder

(2007). Vegetable cultivation in North West Himalayan region: a study of Indian state. Banaras Hindu University - $C A B$ Abstracts International Journal of Agriculture and Biology; 2007. 9(4):602-605.

Yadav, S. M. Rai, J. (2012). Production and economics of major vegetable crops in district Mirzapur (UP) India. Banaras Hindu University - CAB Abstracts Environment and Ecology; 2012. 30(2):336-339.

\section{How to cite this article:}

Punam Kushwaha, Harendra Pratap Singh Choudhri, G.P. Singh, Ashutosh Kumar Ranjan and Abhineet. 2018. A Study on the Farm Asset Structures, Cropping Pattern and Cropping Intensity of Sample Farms in Ghazipur District of Eastern Uttar Pradesh, India. Int.J.Curr.Microbiol.App.Sci. 7(03): 971-978. doi: https://doi.org/10.20546/ijcmas.2018.703.115 